\title{
A Pilot Study on Nanotherapy of Momordica charantia against Trimethyltin Chloride-Induced Neurotoxicity in Danio rerio (Zebrafish)
}

\author{
Bharathi Kumar, ${ }^{1}$ Siva Vijayakumar Tharumasivam, ${ }^{1}$ Vasuki Boominathan, ${ }^{1,2}$ \\ Elumalai Perumal, ${ }^{3}$ Prabu Dhandapani, ${ }^{4}$ Kumaravel Kaliyaperumal, ${ }^{5}$ Suresh Arumugam, \\ Kumaran Subramanian, ${ }^{7}$ Pugazhvendan Sampath Renuga, ${ }^{8,9}$ Vasanth Shakthivel, ${ }^{10}$ \\ Bupesh Giridharan, ${ }^{10}$ Wilson Aruni, ${ }^{11,12}$ and Lokesh Kumar Boopathy $\mathbb{D}^{13}$
}

${ }^{1} P G$ \& Research Department of Biotechnology, Srimad Andavan Arts \& Science College, Srirangam, Tiruchirappalli 620005, India ${ }^{2}$ Department of Biotechnology Bharathidasan Uniersity, Tiruchirappalli 620024, India

${ }^{3}$ Departments of Pharmacology, Saveetha Dental College and Hospital, Chennai, Tamil Nadu, India

${ }^{4}$ Department of Microbiology, Dr. ALM PG IBMS, University of Madras, Chennai, Tamil Nadu, India

${ }^{5}$ National Navel Orange Engineering Research Centre, School of Life Sciences, Gannan Normal University, Ganzhou, Jiangxi, China

${ }^{6}$ Central Research Laboratory, Meenakshi Medical College Hospital \& Research Institute, Kanchipuram, Tamil Nadu, India

${ }^{7}$ Centre for Drug Discovery and Development, Sathyabama Institute of Science and Technology, Chennai, Tamil Nadu, India

${ }^{8}$ Department of Zoology, Annamalai University, Annamalai Nagar, Cuddalore, 608002 Tamil Nadu, India

${ }^{9}$ Department of Zoology, Arignar Anna Government Arts College, Cheyyar, 604407 Tamil Nadu, India

${ }^{10}$ Department of Forest Science, Nagaland Univeristy, Nagaland 798627, India

${ }^{11}$ Department of Biotechnology, School of Bio and Chemical Engineering, Sathyabama Institute of Science and Technology, Chennai, Tamil Nadu, India

${ }^{12}$ School of Medicine, Loma Linda University, CA 92354, USA

${ }^{13}$ College of Health Sciences, Mizan Tepi University, Mizan Teferi, Ethiopia

Correspondence should be addressed to Lokesh Kumar Boopathy; lokeshkumarunom@gmail.com

Received 27 August 2021; Accepted 4 October 2021; Published 9 November 2021

Academic Editor: Mahendran Vanaja

Copyright (c) 2021 Bharathi Kumar et al. This is an open access article distributed under the Creative Commons Attribution License, which permits unrestricted use, distribution, and reproduction in any medium, provided the original work is properly cited.

Background. The direct or indirect effect of chemicals on the nervous system of humans or animals is referred to as neurotoxicity. Trimethyltin chloride (TMT) intoxication causes behavioral and cognitive deficiencies in humans and experimental animals. TMT has long been used as a model toxicant in the study of central nervous system (CNS) toxicity. Momordica charantia, which is used in traditional herbal medicine, has a variety of pharmacological functions. Mesoporous silica nanoparticles have a higher loading capacity, are less dense, and have a larger specific area. Objectives. To investigate a possible nanotherapy for Alzheimer's disease caused by trimethyltin chloride in freshwater zebrafish. Methods. An aqueous extract of M.charantia was used to perform the primary and secondary screening. The DPPH (2,2-diphenyl-1-picrylhydrazyl) radical scavenging assay was used to determine the antioxidant capacity of crude aqueous extracts of $M$. charantia. Mesoporous silica nanoparticles are made using a CTAB surfactant chemical process and tetraethyl orthosilicate. UV-Vis spectroscopy, Fourier transform infrared spectroscopy, scanning electron microscope, and EDAX were used to characterize it. Danio rerio was used to test the trimethyltin chloride for Alzheimer's disease. The M. charantia and mesoporous silica nanoparticles were then tested in the same method. Results. The extract has no adverse effects on zebrafish, indicating that $M$. charantia is safe for human consumption. The histopathological findings indicate that the tissues of the fish infected with the extract had no pathological modifications. Conclusion. The M. charantia showed higher antioxidant activity and anticholinesterase activity, and upon further characterization and assessment, this could be a safe and potential drug candidate for Neurotoxicity. 


\section{Introduction}

Trimethyltin chloride (TMT) has been found in drinking water sources, household water systems, marine environments, and aquatic specimens [1]. Between 1978 and 2008, TMT poisoned 1849 people in 67 deaths around the world. The most frequent acute TMT exposures tend to be environmental, occurring during plastic manufacturing or other industrial processes involving plastic heating. Ingestion of organotin-contaminated food or water in places where plastics manufacture is prevalent is also expected to become a growing concern [2]. TMT intoxication causes severe behavioral and neurological problems in humans and laboratory animals. A limbic-cerebellar syndrome is the most common neurotoxic presentation in human cases, with symptoms such as memory loss, confusion, epilepsy, tinnitus, insomnia, and depression [3]. TMT has long been used as a model toxicant in the study of CNS toxicity. Experiments in the marine fish Sebastiscus marmoratus have led to suggestions that the glutamatergic receptor NMDAR and its signaling pathway components are essential for TMT neurotoxicity [4]. Neurotoxicity is a dynamic condition that is difficult to be successfully treated with a single treatment or other intervention. Current strategies are aimed at assisting patients in maintaining brain function, managing behavioral effects, and slowing the progression of disease symptoms. Cholinesterase inhibitors are not widely used in allopathic medicine, and existing therapies may not result in enough acetylcholine activity to assist with neurotoxicity control. The study of natural compounds with antioxidative and antiaging properties that may also be beneficial for neurodegenerative diseases has evolved into the area of phytochemicals. Donepezil is a cholinesterase agent that inhibits the degradation of acetylcholine in the brain. It is used to relieve effects in mild, moderate, and extreme cases. Various plants have been used as medicine all over the world since ancient times. For thousands of years, Momordica charantia (Momordica species) has been popular as a medicine and a vegetable. $M$. charania is a Cucurbitaceae plant that is widely known as bitter gourd, balsam pear, or bitter melon. The fruit of $M$. charantia is a thin cucumber-like spindle with pimples on the surface; the young fruit is emerald green and becomes orange when ripe, while the flesh becomes scarlet from white when it matures. The fruit can be eaten at any point of its growth, and it is widely consumed as a vegetable in various regions of the globe [5]. The whole plant, especially the seeds and fruit, has considerable pharmacological effects, for example, it has been used in the treatment of diabetes since ancient times and continues to play an important role in diabetes prevention and treatment in many developing countries. This plant is used in conventional herbal medicine and has several pharmacological properties, including antidiabetic, abortifacient, anthelmintic, contraceptive, antimalarial, and laxative properties. Dysmenorrhea, eczema, gout, jaundice, leprosy, piles, measles, psoriasis, rheumatism, and scabies are all treated by it [5]. The future applications of mesoporous silica nanoparticles (MSNs) have piqued the interest of many scientists over the last decade. Because of their higher loading volume, lower density, and the larger specific area, hollow mesoporous silica nanoparticles (HMSNs) with a deep central hole and an external mesoporous silica shell provide additional benefits.
TABle 1: Phytochemical analysis of $M$. charantia.

\begin{tabular}{lcc}
\hline S. No. & Phytochemical tests & Results \\
\hline 1 & Test for steroid & Present \\
2 & Test for alkane & Present \\
3 & Test for flavonoids & Present \\
4 & Test for saponins & Present \\
5 & Test for terpenoids & Present \\
6 & Test for phenols & Absent \\
7 & Test for cocumarins & Present \\
8 & Test for emodols & Absent \\
9 & Test for phlobatannis & Absent \\
10 & Test for phlobatannis & Present \\
11 & Test for phlobatannis & Absent \\
12 & Test for phytosterol & Present \\
13 & Test for reducing compound & Present \\
14 & Test for cardiac glycosides & Absent \\
\hline
\end{tabular}

Mesoporous silica nanoparticles due to their low toxicity and high drug loading capacity so are used in controlled and target drug delivery systems. Silica is widely present in the environment in comparison to other metal oxides like titanium and iron oxides, and it has comparatively better biocompatibility [6]. The mesoporous form of silica has unique properties, particularly in the loading of therapeutic agents at high quantities, and in the subsequent releases. Due to the strong Si-O bond, silica-based mesoporous nanoparticles are more stable to external responses such as degradation and mechanical stress as compared to niosomes, liposomes, and dendrimers which inhibit the need for any external stabilization in the synthesis of MSNs [4, 7]. The mesoporous structure such as pore size and porosity can be tuned to the size and type of drugs.

Furthermore, MSN is very effective in a variety of applications, including enzyme encapsulation, drug transport and delivery, reactive material packaging, and light emitting. However, they have received little attention as corrosion inhibitor nanocarriers, with just a few studies conducted on them [8]. The zebrafish (Danio rerio) belongs to the minnow family of freshwater fish. Cypriniformes (Cyprinidae) is a family of fish in the class Cypriniformes. The zebrafish (Danio rerio) is an important model organism for vertebrate evolution, genetics, and human biology and disease, as well as a common tropical fish pet. Danio rerio has many characteristics, including large family size, external growth, and low maintenance and production expense [9].

This research is aimed at investigating a possible nanotherapy for neurotoxicity induced by trimethyltin chloride in freshwater Danio rerio (zebrafish).

\section{Materials and Methods}

2.1. Chemicals. Tetraethyl orthosilicate $(100 \mathrm{ml})$ was purchased from the Sigma Industry, and trimethyltinchloride was purchased in TCI Chemicals $1 \mathrm{~g}$ (India) Pvt. Ltd. 


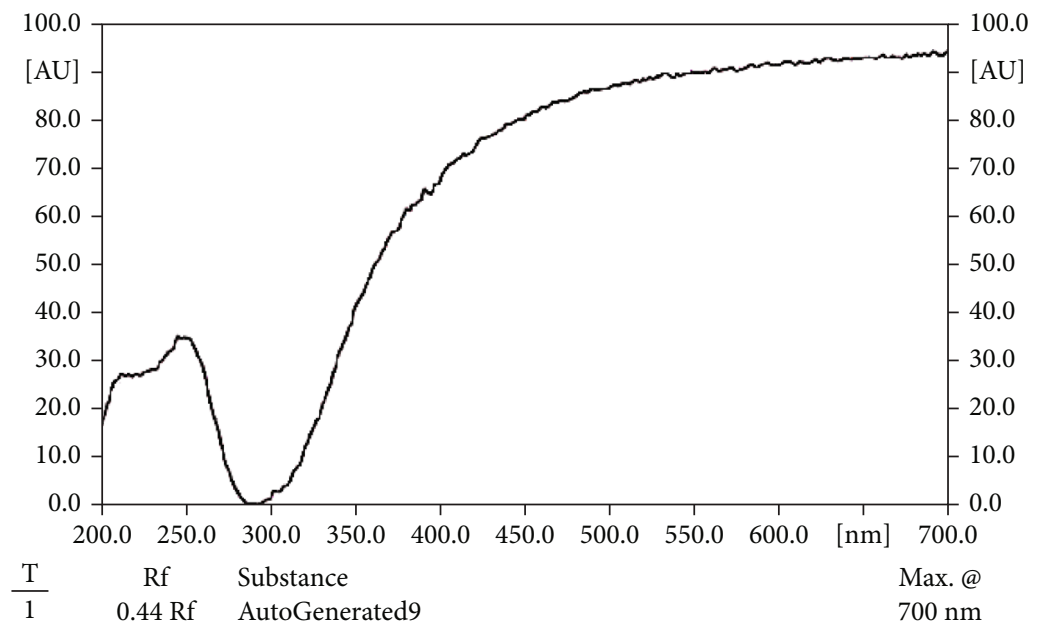

(a)

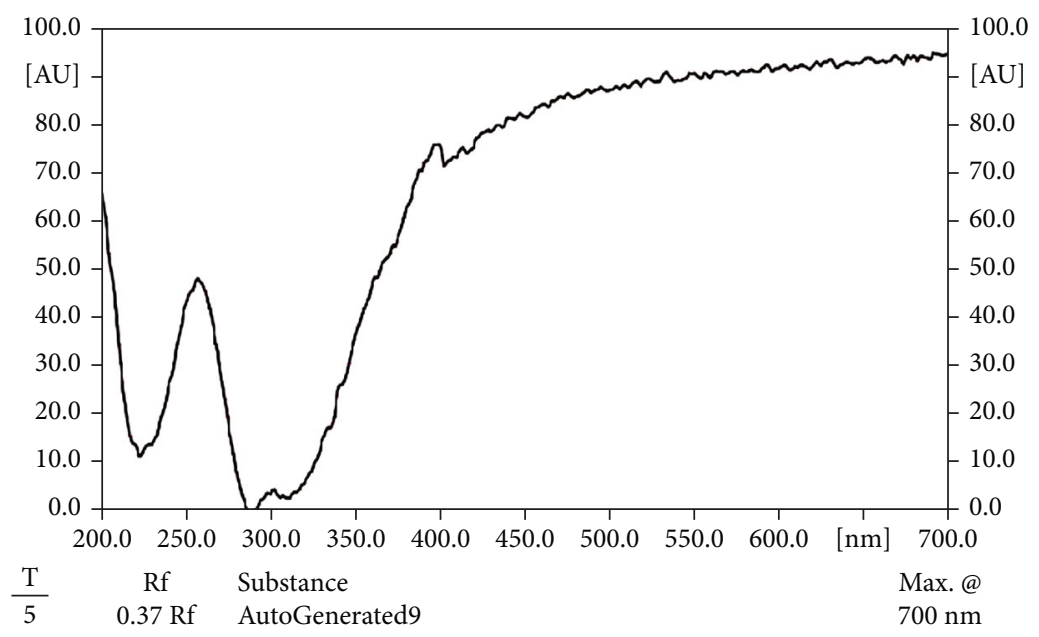

(b)

FigURE 1: (a) Graph of stigmasterol. (b) Graph of lupeol.

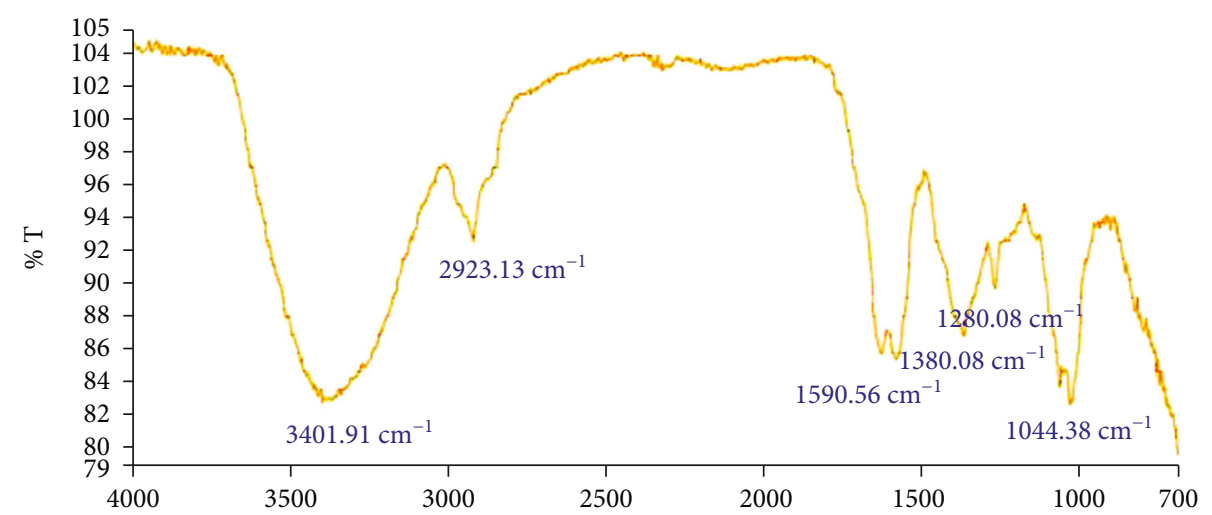

FIGURE 2: Fourier transform-infrared (FT-IR) spectrum of $M$. charantia extract. 


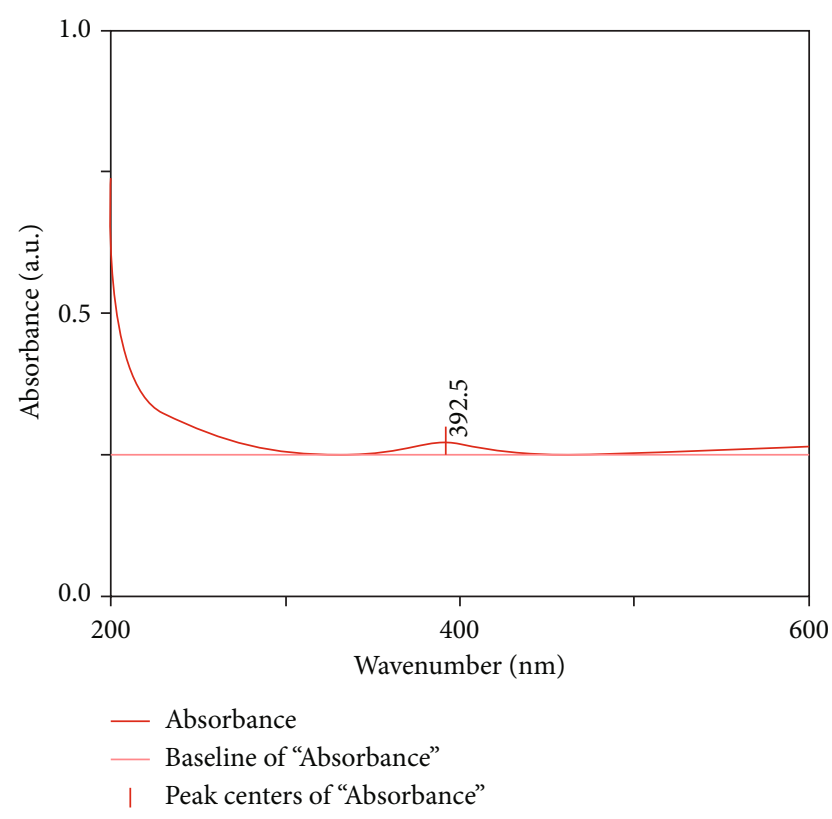

FIGURE 3: UV-visible spectrophotometer analysis of mesoporous silica nanoparticle.

2.2. Collection and Authentication of the Sample (Momordica charantia). M. charantia fruit samples were collected from a local farming field at Ayyarmalai village near Karur, Tamil Nadu. The M. charantia was air-dried and pulverized, sieved to get a coarse powder, and stored in airproof containers until their experimental use. The collected fruit was authenticated from the department of Botany, St.Joseph's College, Trichy, with a voucher specimen submitted and got the reference number of K.B.001.

\subsection{Extraction of M. charantia (Cold Extraction). M. charan-} tia powder was mixed with a suitable amount of ethanol (95\%), extracted in an orbital shaker for $72 \mathrm{~h}$, and then filtered through muslin cloth and dry extraction by using a vacuum.

2.4. Preliminary Screening of Phytochemical Analysis. The ethanol extract was assessed for the existence of the phytochemical analysis by using the following standard method [9].

\subsection{High-Performance Thin Layer (HPTLC) Identification.} The solution was packed with $95 \%$ ethanol. The stationary phase was a silica gel-coated sheet, and the mobile phase was ethyl acetate: methanol: distilled water $75: 15: 10$ ratios. The $\mathrm{Rf}$ was observed as a violet spot [6].

2.6. Fourier Transform-Infrared (FT-IR) Spectrum Analysis of M. charantia Extract. FT-IR was used to record the infrared spectrum (Shimadzu). To make the pellet samples, the M.charantia sample and $\mathrm{KBr}$ were mixed in a $1: 10$ ratio in a $\mathrm{KBr}$ press. The spectra were recorded at wavenumbers ranging from 4000 to $400 \mathrm{~cm}^{-1}$ [7].

2.7. Synthesis of Mesoporous Silica Nanoparticle. To reach a $\mathrm{pH}$ of 12.3 , cetyltrimethylammonium bromide $(0.5 \mathrm{~g})$,
2.0 M NaOH $(1.75 \mathrm{ml})$, and deionized water $(120 \mathrm{~g})$ were heated at $80^{\circ} \mathrm{C}$ for 30 minutes. $2.5 \mathrm{ml}$ of TEOS (tetraethyl orthosilicate) is dissolved into this transparent solution under intense stirring. Within three minutes of stirring at $550 \mathrm{rpm}$ after the injection, white precipitation is noticeable. For 2 hours, the reaction temperature is maintained at $80^{\circ} \mathrm{C}$. Hot filtration is used to isolate the product, which is then washed with a large volume of water and methanol $(5 \mathrm{ml}$ every three times). At $60^{\circ} \mathrm{C}$ for 6 hours, an acid extraction was carried out in methanol $(100 \mathrm{~mL})$ with a mixture of concentrated hydrochloric acid $(1.0 \mathrm{~mL})$ and as-made materials $(1.0 \mathrm{~g})$. The solid products that had been separated from the surfactant were filtered, washed with water and methanol and then dried under a vacuum.

\section{Characterization of Nanoparticle}

3.1. UV-Vis Spectroscopy. A $1.2 \mathrm{mg}$ sample was collected after sample preparation and subjected to UV-Vis spectroscopy. A miniature fiber optic spectrometer was used to record UV-Vis spectra using versatile lamps optimized for the visible-near infrared Vis-NIR $(200-500 \mathrm{~nm})$. The samples had been diluted 100 times before being measured. Averaging 1000 single observations yielded the findings described here [6].

3.2. Fourier Transform-Infrared Spectroscopy Analysis of Mesoporous Silica Nanoparticle. After sample preparation and Fourier transform infrared spectroscopy, a sample of mesoporous silica nanoparticle $0.5 \mathrm{mg}$ was taken (FT-IR). The vibrational stretch frequency of metal-oxygen bonds is revealed by Fourier transform infrared spectroscopy. A continuum source of light is used to emit light over a wide range of infrared wavelengths for Fourier transform infrared spectroscopy. Light commuting electrons are excited from their ground state to an excited state as an atom or molecule absorbs energy. The atoms in a molecule will vibrate and rotate in response to one another. These vibrations and rotations also have discrete energy levels, which can be considered as being packed on top of each electronic level. The absorption of visible or UV radiation occurs to the excitation of outer electrons [7].

3.3. Scanning Electron Microscopy with EDAX. Sample of mesoporous silica nanoparticle $1 \mathrm{~g}$ was taken after preparation of sample and scanning electron microscope. The electron beam used to probe the sample is produced by the electron gun in a scanning electron microscope. Electrons are emitted from a cathode, accelerated by electrical fields, and focused on the source's first optical image. The size and shape of the apparent source, beam acceleration, and current are the primary determinants of a scanning electron microscope's performance and resolution. Spot welded to metal posts is a bent tungsten wire filament with a diameter of around 100 micrometers. These posts are embedded in a ceramic holder and extend out the other side to provide electrical connections. In operation, the filament will be heated by passing an electrical current through it. The optimum filament temperature for the thermionic emission of electrons 


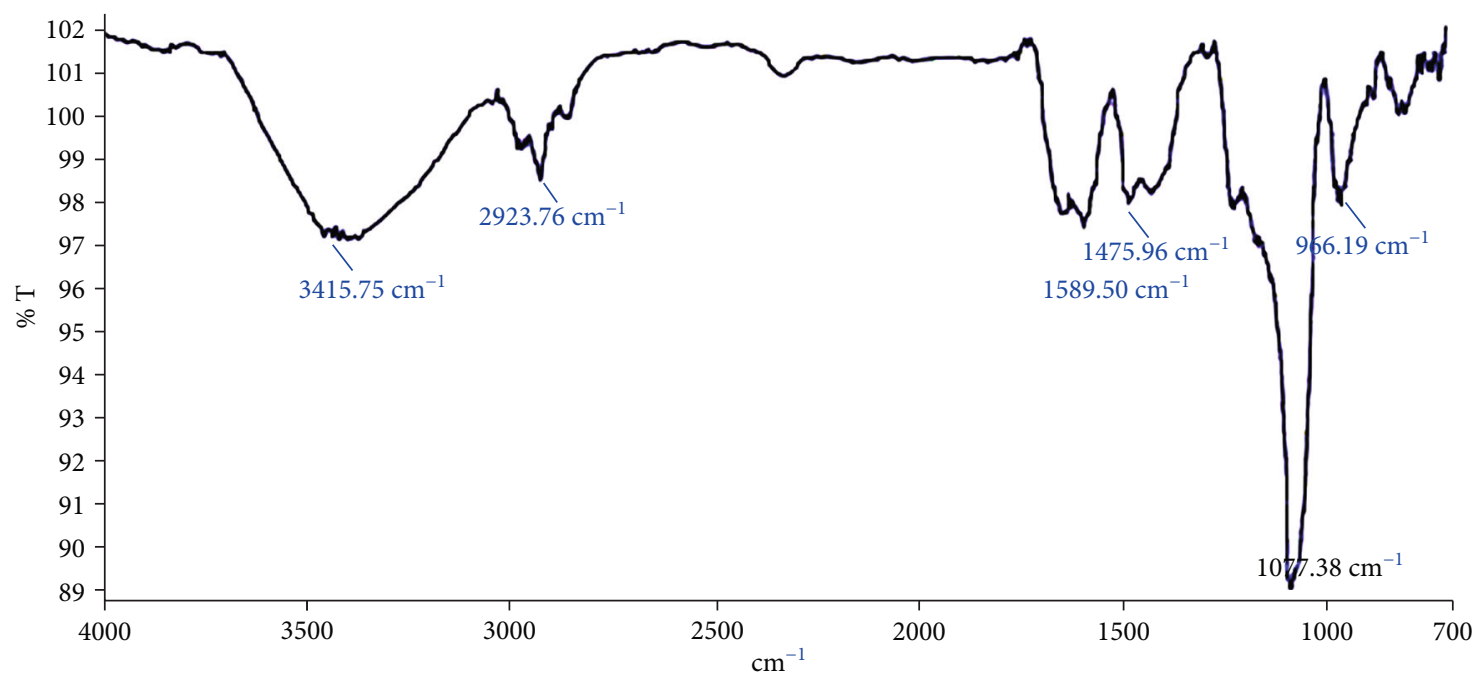

FIGURE 4: Fourier transform-infrared (FT-IR) spectrum of mesoporous silica.

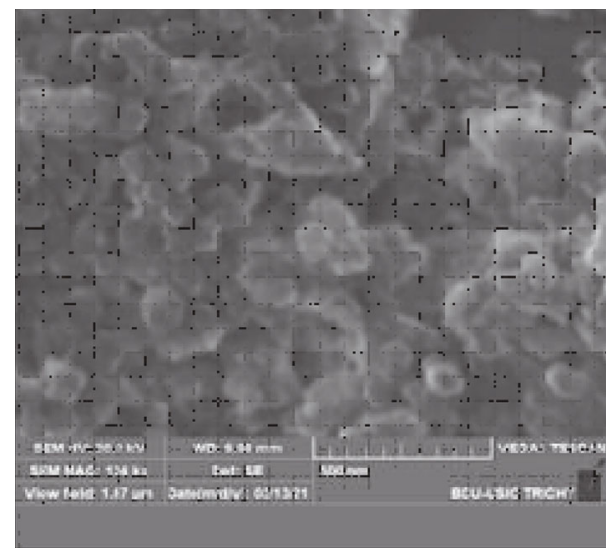

(a)

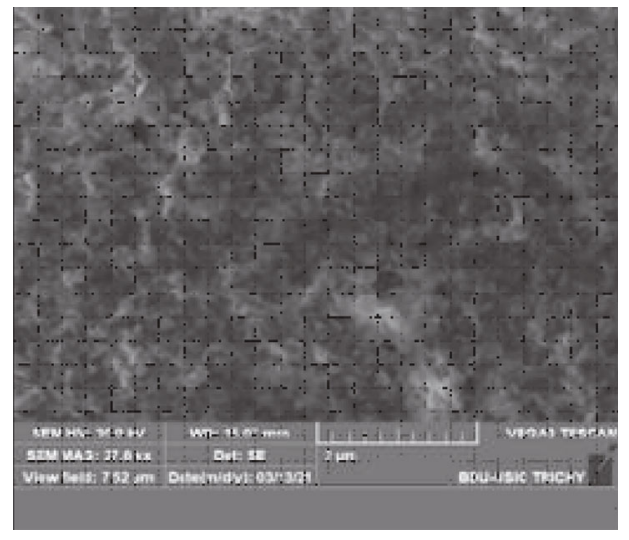

(b)

Figure 5: $(\mathrm{a}, \mathrm{b})$ Scanning electron microscopy analysis of mesoporous silica nanoparticle.

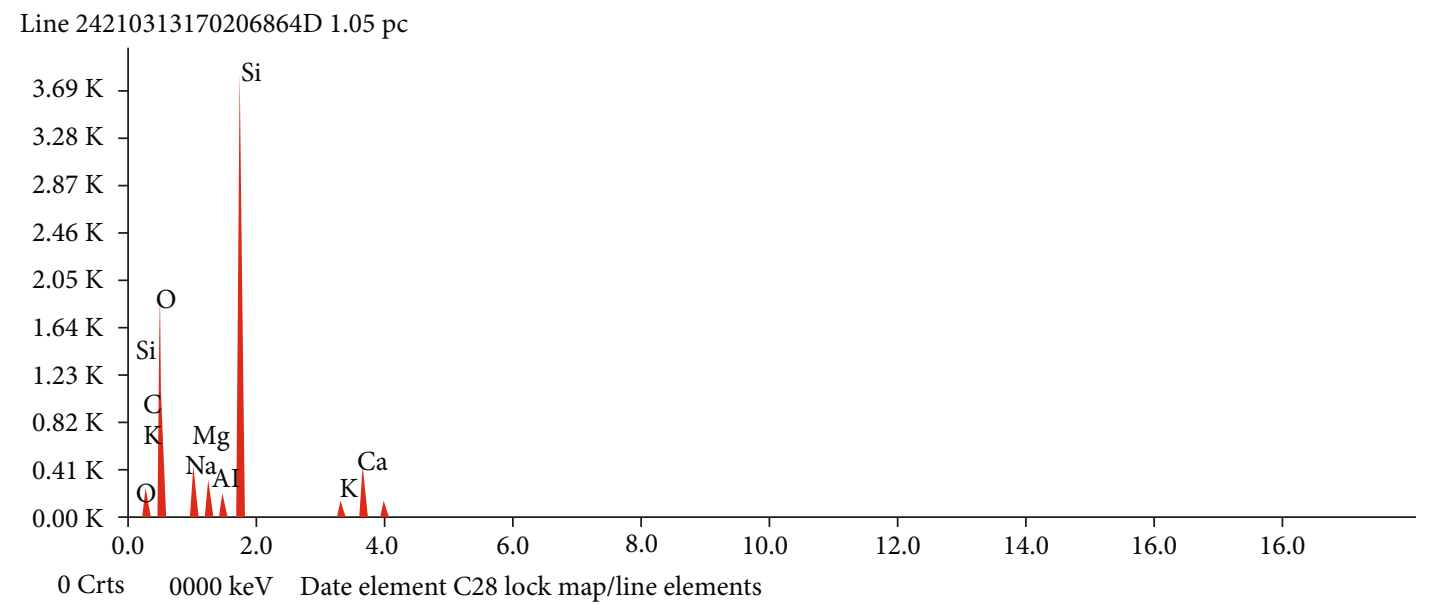

FIGURE 6: EDS analysis of synthesized mesoporous silica nanoparticles. 


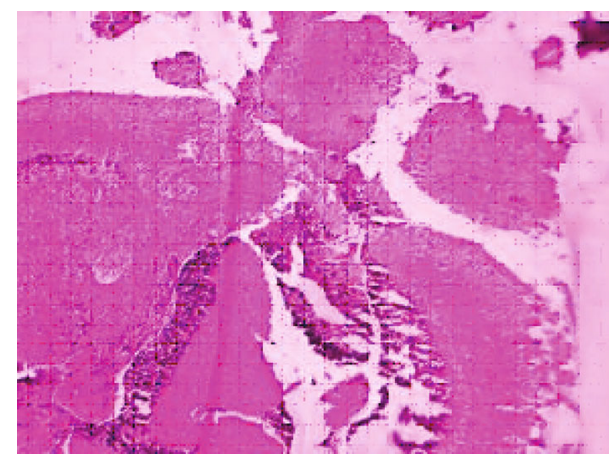

Figure 7: (a) Brain-group I (control) (healthy).

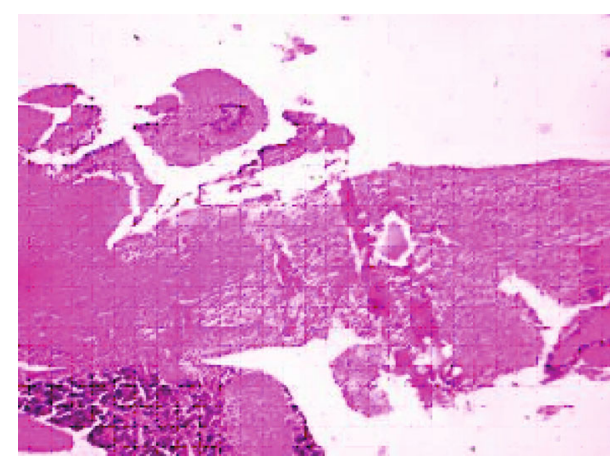

Figure 8: (b) Group 2: brain treated damaged (day 1: trimethyltin chloride).

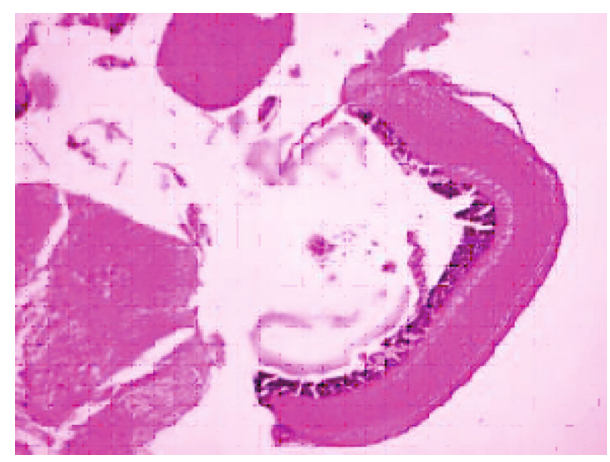

FIGURE 9: (c) Group 3: brain treated (day 2: trimethyltin chloride).

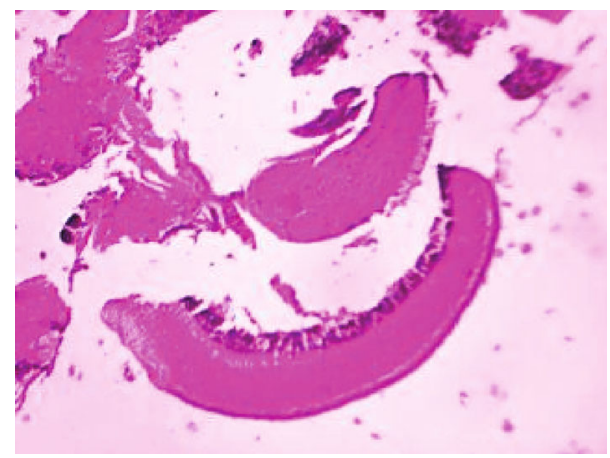

FIGURE 10: (d) Group 4: brain treated [trimethyltin chloride + FDA approved drug (donepezil)].

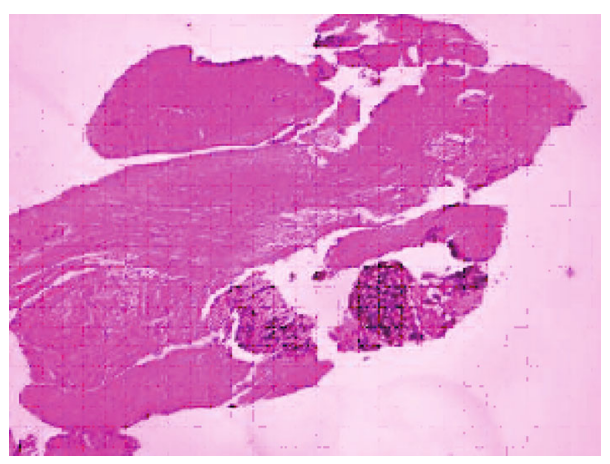

FIGURE 11: (e) Group 5: brain treated (trimethyltin chloride + charantin).

is around $2700 \mathrm{~K}$. The accelerating voltage, generally, between -500 volts and $-50,000$ volts DC, is applied to the Wehnelt cylinder. Energy dispersive X-ray analysis (EDX), referred to as EDS or EDAX, is an X-ray technique used to identify the elemental composition of materials.

3.4. Trimethyltin Chloride. Two micromolar solutions of trimethyltin chloride are prepared by adding $0.0003 \mathrm{~g}$ in $1000 \mathrm{ml}$ water. $1 \mathrm{~h}$ before the experiment, the zebrafish was directly placed in the above trimethyltin chloride solution.

3.5. Donepezil. Donepezil $1.5 \mathrm{mg}$ was dissolved in $1 \mathrm{~mL}$ of water. $2.5 \mu \mathrm{L}$ is pipetted out into an Eppendorf tube, and $25 \mu \mathrm{L}$ water was added to it. This solution contains $3.75 \mu \mathrm{g}$ of donepezil in $25 \mu \mathrm{L}$ water. From the above solution, $5 \mu \mathrm{L}$ is sucked into a pipette and directly administered to zebrafish feed, where $5 \mu \mathrm{L}$ contains $0.75 \mu \mathrm{g}$ of donepezil.

3.6. Model Animals. Adult zebrafish (Danio rario) weighing $0.5-1.0 \mathrm{~g}$ is procured from a local aquarium pet store. They were left in housing tanks with water at a density of 5 animals per $3 \mathrm{~L}$ at $14-10 \mathrm{~h}$ light and dark photoperiod with continuous aeration and pellet diet for about 10 days before the commencement of the experiment.

3.7. Cell Viability Test. MTT assay can be used to determine the extract's cytotoxic activity. In a summary, 104 murine macrophage cell line RAW 264.7 cells per well were seeded in a 96-well microplate and treated for 72 hours with varying concentrations of $M$. charantia extracts. $15 \mu \mathrm{l}$ of MTT will be added at the end of the treatment period and incubated at $37^{\circ} \mathrm{C}$ for 4 hours. The formazan products will be dissolved in $150 \mu \mathrm{l}$ of DMSO, and their absorbancxe will be measured at $370 \mathrm{~nm}$ [10]. The percent cell cytotoxicity will be calculated using the formula:

$\%$ Cytotoxicity $=100-\{$ O.D control - O.D test $\times 100 / O . D$ control

3.8. Drug Loading on Mesoporous Silica Nanoparticle. Drug loading on mesoporous silica nanoparticles dissolves $100 \mathrm{mg}$ of the momordica charanthin drug in ethanol $(1 \mathrm{ml})$. Add $100 \mathrm{mg}$ of MSNP to the clear solution of the 


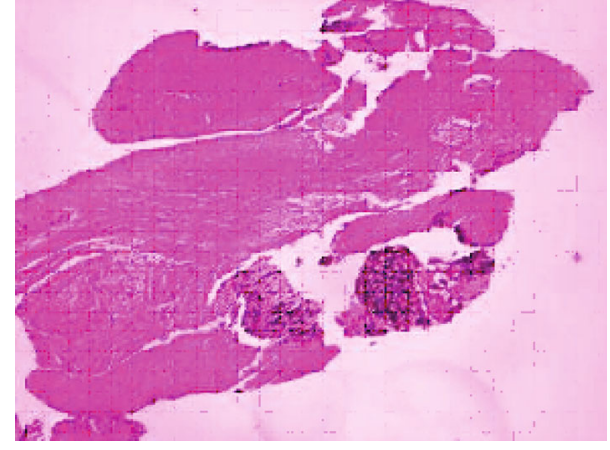

Figure 12: (f) Group 6: brain treated [trimethyltin chloride + (charantin + mesoporous silica nanoparticle)].

drug and the solvent. Stir the sample and MSNP mixture at $300 \mathrm{rpm}$ for 24 hours [10].

3.9. In Vivo Method Group of Fish (Danio rario). This is a list of groups in untreated fish (Danio rario).

Group 1-control.

This is a list of groups in treated fish (Danio rario).

Group 2-(day 1) trimethyltin chloride.

Group 3-(day 2) trimethyltin chloride.

Group 4-trimethyltin chloride + FDA approved drug (donepezil).

Group 5-trimethyltin chloride + charantin.

Group 6-trimethyltin chloride + (charantin + mesoporous silica nanoparticle).

Histological examinations were carried out in the way described by [11], with a few modifications. Following fixation, the samples were dehydrated, embedded in paraffin wax, sliced at 3-5 $\mu \mathrm{m}$ with a microtome, and stained with hematoxylin and eosin. DPX mountant was used to make the stained slides permanent for microscopic examinations after staining. The slides were blinded to the observer, and all serial sections were scored histopathologically. Previously, whole fish sections of untreated zebrafish were used to determine zebrafish histology. The slides were examined under a $\times 40$ microscope.

\section{Neurochemical and Antioxidant Estimations}

4.1. Acetylcholinesterase Activity Assay. The AChE was made from the brain homogenate of 30 fishes, centrifuged, and the supernatant was collected and used as an enzyme source for the AChE assay. Differential concentrations (100$500 \mathrm{mg} / \mathrm{mL}$ ) of aqueous extracts of $M$. charantia were incubated for 45 minutes at room temperature with $50 \mu \mathrm{l}$ of AChE makeup and $200 \mu \mathrm{l}$ of tris-HCL. $125 \mu \mathrm{l}$ of $3 \mathrm{mM}$ DTNB was applied to the reagent mixtures, and the total volume was increased to $650 \mu \mathrm{l}$ with tris- $\mathrm{HCl}$ buffer ( $\mathrm{pH}$ 8.0). To initiate enzyme activity, $1 \mathrm{mM}$ ATCI was added to $25 \mu \mathrm{l}$ of the reaction mixture. A UV-visible spectrophotometer was used to detect the formation of 5-di-this-2nitrobenzoic acid anion, and the absorbance was measured at $405 \mathrm{~nm}$. The tests were performed in triplicate. The medication donepezil was used as a standard [12].
4.2. DPPH (2, 2-Diphenyl-1-Picrylhydrazyl) Assay. To prepare the stock solution, $1.97 \mathrm{~g}$ of $\mathrm{DPPH}$ was dissolved in $50 \mathrm{~mL}$ of methanol. Various concentrations of M.charantia aqueous extract were analyzed using a dilution process. $100 \mathrm{mg}-500 \mathrm{mg}$ extracts were used in $10 \mathrm{mg}$ in $1 \mathrm{~mL}$ water, and their various concentrations were prepared. The mixture was shaken and sets it aside for 30 minutes at room temperature. Then, at $517 \mathrm{~nm}$, the absorbance was measured. All of the tests were performed in triplicates with ascorbic acid as the standard [13].

4.3. Assay of Total Reducing Power. Various concentrations of $M$. charantia fruit ethanol extract $(10 \mathrm{mg} / 1 \mathrm{~mL})$ were combined with phosphate buffer $(0.5 \mathrm{~mL}, 0.2 \mathrm{M}, \mathrm{pH}$ 6) and potassium ferricyanide $\left[\mathrm{K}_{3} \mathrm{Fe}(\mathrm{CN}) 6\right]$ in $1 \mathrm{~mL}$ of distilled water $(0.5 \mathrm{~mL}, 1$ percent). For 20 minutes, the mixture was incubated at $50^{\circ} \mathrm{C}$. Trichloroacetic acid (10\%) aliquots $(0.5 \mathrm{~mL})$ were added to the mixture, which was then centrifuged for 10 minutes at $2500 \mathrm{rpm}$. The absorbance of the upper layer of solution $(0.5 \mathrm{~mL})$ was measured in a spectrophotometer at $700 \mathrm{~nm}$ using distilled water $(0.5 \mathrm{~mL})$ and ferric chloride $\mathrm{FeCl}_{3}(0.5 \mathrm{~mL}, 0.1 \%)$. Increased reaction mixture absorbance indicates increased reducing power.

4.4. Assay of Lipid Peroxidation. The peroxidation of lipids was measured using thiobarbituric acid reactive substances. The brain homogenate $(10 \mathrm{ml})$ was mixed with $150 \mathrm{ml}$ of acetic acid (20\%), $150 \mathrm{ml}$ of thiobarbituric acid (0.8\%), $20 \mathrm{ml}$ of sodium dodecyl sulfate $(8.1 \%)$, and $50 \mathrm{~mL}$ of distilled water (reaction mixture); heated for $30 \mathrm{~min}$ at $95.8^{\circ} \mathrm{C}$; and then cooled and centrifuged $(3000 \mathrm{~g}$ during $10 \mathrm{~min}$ at $15^{\circ} \mathrm{C}$ ) by adding $100 \mathrm{ml}$ of water and $500 \mathrm{ml}$ of $\mathrm{n}$-butanol. The fluorescence of the supernatant organic phase $(150 \mathrm{ml})$ was calculated in a microplate reader at the wavenumbers $515 \mathrm{~nm}-553 \mathrm{~nm}$. The concentration of TBARS was determined using the standard tetra methoxy propane (TMP).

4.5. Assay of Total Antioxidant. The ability of the sample to reduce ferric tripridytriazine (Fe (II)-TPTZ) complex to intense blue-colored ferrous tripyridyltriazine (Fe (II)TPTZ, which is read at $593 \mathrm{~nm}$, is required for the FRAP assay. First, $1.5 \mathrm{ml}$ of freshly prepared FRAP reagent $(25 \mathrm{ml}$ of $300 \mathrm{mM} / \mathrm{L}$ acetate buffer $\mathrm{pH} 3,2.5 \mathrm{ml}$ of $10 \mathrm{mM} / \mathrm{L}$ TPTZ in $40 \mathrm{mM} / \mathrm{L} \mathrm{HCl}, 20 \mathrm{mM} / \mathrm{L}$ ferric chloride solution) is mixed with $50 \mu \mathrm{l}$ of varying concentrations of $M$. charantia (100$500 \mu \mathrm{l})$ extracted successively in different solvents and $150 \mu \mathrm{l}$ of distilled water. At $593 \mathrm{~nm}$, absorbance was measured for 4 minutes (every 10 seconds).

\section{Statistical Analysis}

One-way ANOVA was used for data analysis and presented as a mean \pm sandard error of the mean. Latencies of multiple groups were compared using Dunnett's multiple comparison test.

\section{Results and Discussion}

By exposing the ripe fruits to an orbital shaker, a sample of M.charantia was collected (cold extraction). It was collected 


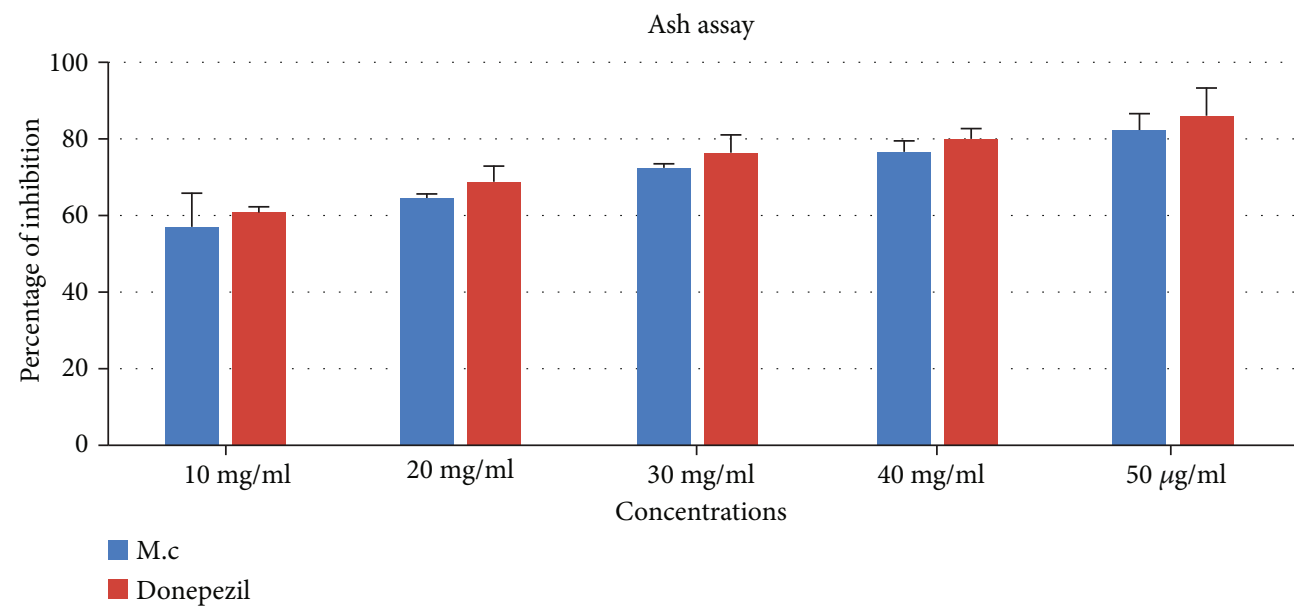

FIgURE 13: Acetylcholinesterase assay (M. charantia).

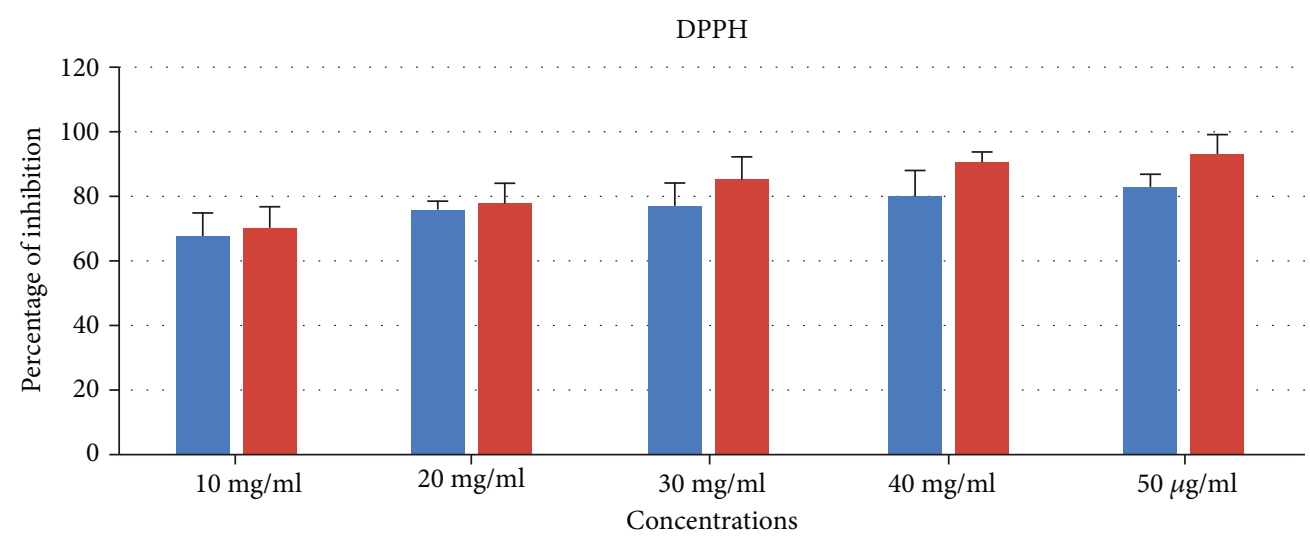

M.c

A. a

FIGURE 14: DPPH (2, 2-diphenyl-1-picrylhydrazyl) radical scavenging activity (M. charantia, A.a: ascorbic acid).

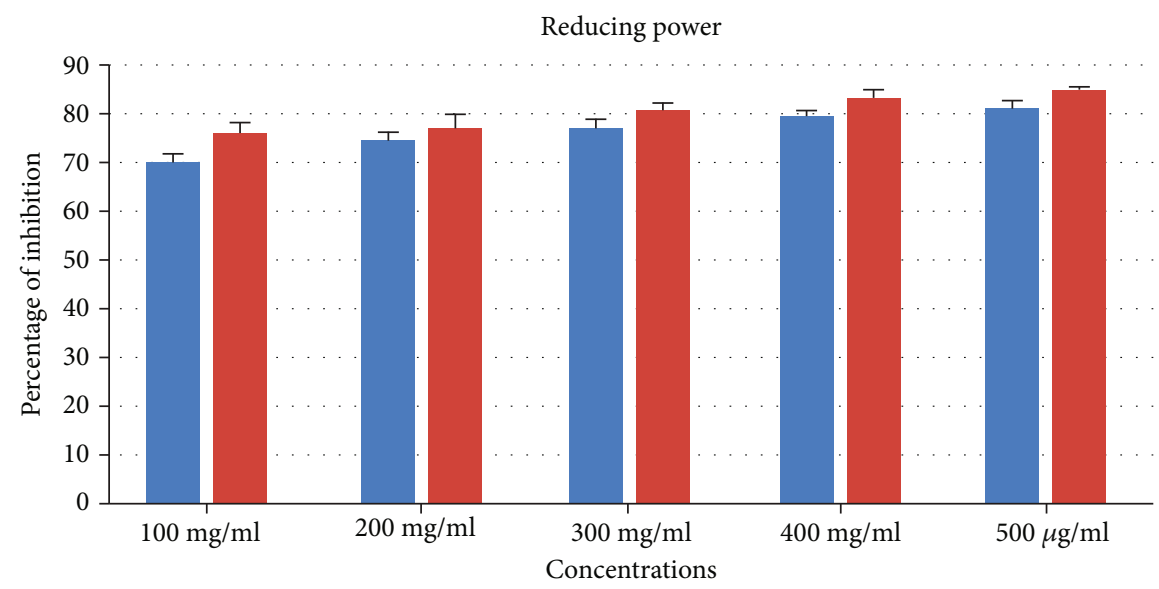

M.c

A.a

Figure 15: Reducing power assay (M. charantia, A.a: ascorbic acid). 


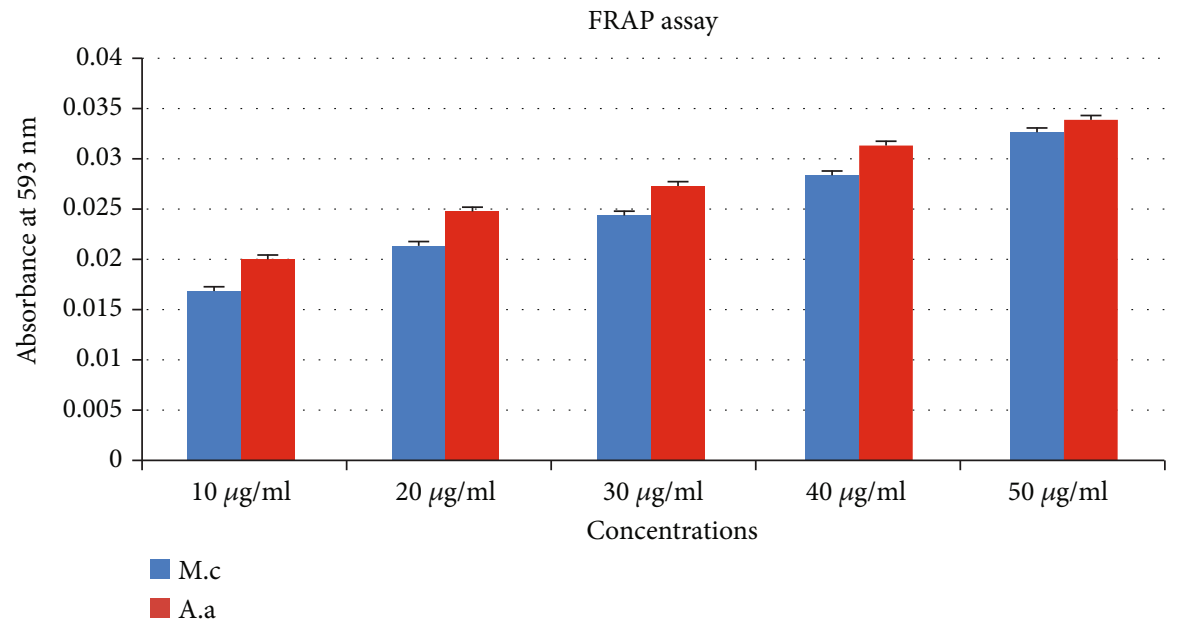

Figure 16: Total antioxidant assay (M. charantia, A.a: - ascorbic acid).

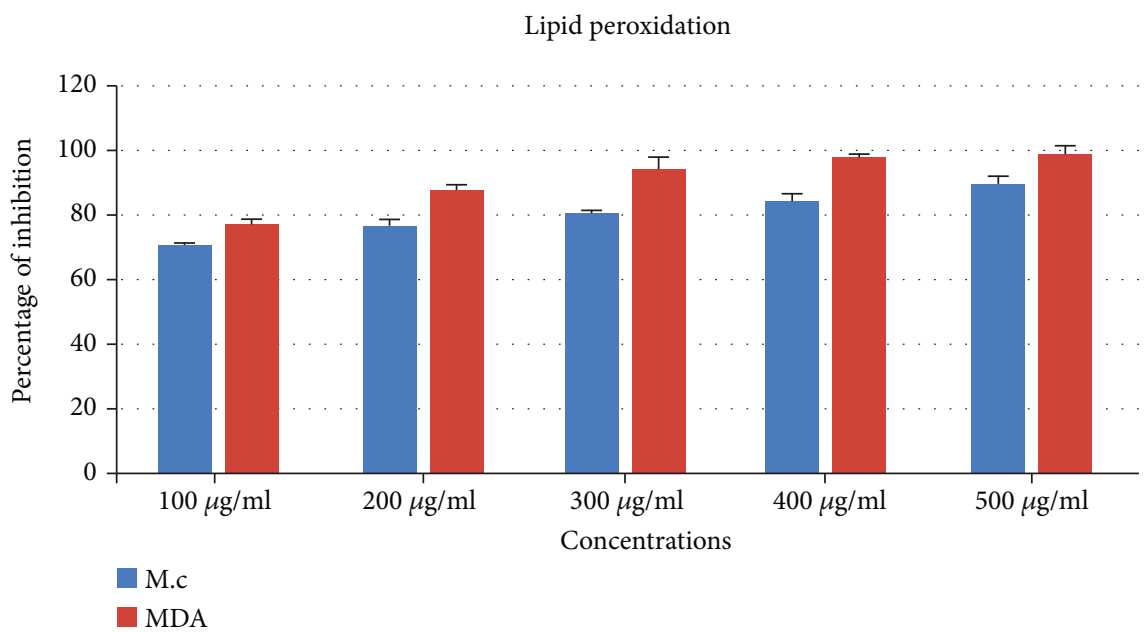

FIgURE 17: Lipid peroxidation assay ( $M$. charantia, A.a: ascorbic acid).

in the form of a brownish yellow colloidal crude. Similar to [14], preliminary phytochemical screening of M.charantia aqueous extract revealed the presence of phytochemicals such as steroids, alkane, flavonoids, saponins, terpenoids, cocumarins, and alkaloids, and reducing compounds were all included in M.charantia ethanol extract (Table 1). Phenols, emodols, photobatanins, tannis, and cardiac glycosides were not reported. The collected findings can be used as a starting point for identifying bioactive compounds in M.charantia ethanolic extract.

According to the results of High-Performance ThinLayer (HPTLC) Identification, the $\mathrm{Rf}$ value of 0.44 at $700 \mathrm{~nm}$ reveals that the compound was stigmasterol in Figure 1(a), while the Rf value of 0.37 at $700 \mathrm{~nm}$ verifies that the compound was lupeol in Figure 1(b).

The unmodified $M$. charantia FTIR spectrum (Figure 2) is surprisingly clear and well assigned. Methylene $(>\mathrm{CH} 2)$ was assigned to the bands at $2923.13 \mathrm{~cm}-1$. At $3401.91 \mathrm{~cm}$, the hydroxy group $\mathrm{H}$-bonded $\mathrm{OH}$ vibrations can be seen. The $\mathrm{C}=\mathrm{C}-\mathrm{C}$ aromatic ring stretch peaks at $1590.56 \mathrm{~cm}^{-1}$, the $\mathrm{C}-\mathrm{N}$ primary amine peaks at $1044.38 \mathrm{~cm}^{-1}$, and the aro- matic secondary amine C-N stretch peaks at $1280.08 \mathrm{~cm}-1$. The FTIR results indicate similar $\mathrm{O}-\mathrm{H}$ vibrations and peak area values, according to previous findings [15].

6.1. Synthesis of Mesoporous Silica Nanoparticle. The formation of white shading precipitation after applying TEOS (Tetra Ethylene Ortho Silica) to the CTAB surfactant arrangement while nonstop blending confirmed the synthesis of mesoporous silica nanoparticles using the CTAB surfactant process. The MSNs are mixed at a low surfactant concentration to ensure that the organized mesophases depend heavily on the cationic surfactant's cooperation with the forming anionic oligomer orthosilicic corrosive, limiting the size of the mesophases collected. Micrometer-sized particles with circularly requested mesopores were the primary material detailed by versatile analysts.

\subsection{Characterization of Nanoparticle}

6.2.1. UV-Visible Spectrophotometer. The prepared mesoporous silica nanoparticles were characterized using a UV- 
visible spectrophotometer, which reported a peak at $392.5 \mathrm{~nm}$ (Figure 3). This is likely due to the amino group found in silica nanoparticles, in similar study led by (Francesco Armetta et al., 2020). In the present study, the highest absorbance peak was observed within 200-500 nm on the UV-visible spectrophotometer, which agrees with the literature that nanoparticles were synthesized. The results of the FTIR spectrum of the mesoporous silica nanoparticle are further supported by this observation from the UV-visible spectrophotometer.

6.2.2. Fourier Transform-Infrared (FT-IR) Spectrum Analysis of Mesoporous Silica Nanoparticle. The Si-O stretching and asymmetric stretching vibrations bands at $1077.38 \mathrm{~cm}^{-1}$ correspond to silica, respectively (Figure 4). Because of the presence of surfactant templates, the symmetric and asymmetric $\mathrm{C}-\mathrm{H}$ motions can be seen at $2923.76 \mathrm{~cm}^{-1}$. However, after removing the templates, these peaks disappeared, indicating that the surfactants had been removed, and a mesoporous structure had been formed, as seen in Figure 4. N-H stretch aromatic primary amine has a peak at $3415.75 \mathrm{~cm}^{-1},>\mathrm{N}-\mathrm{H}$ secondary amine has a peak at $1588.56 \mathrm{~cm}^{-1}, \mathrm{C}-\mathrm{H}$ aromatic $\mathrm{C}-\mathrm{H}$ in pane bend has a peak at $956.19 \mathrm{~cm}^{-1}$, and methylene $\mathrm{C}-\mathrm{H}$ bend has a peak at $1475.96 \mathrm{~cm}^{-1}$. The FTIR results suggest similar $\mathrm{C}-\mathrm{H}$ vibrations and peak area values, according to prior studies [16].

6.2.3. Scanning Electron Microscopy Analysis of Mesoporous Silica Nanoparticles. A silica nanoparticle is a novel type of mesoporous silica nanomaterial that has a hollow spherical shape. Figures 5(a) and 5(b) show an SEM image of mesoporous silica nanoparticles. Tetraethyl orthosilicate (TEOS) as the silica precursors and cetyl trimethyl ammonium bromide $(\mathrm{CTAB})$ as the pore-forming agent were used to make mesoporous silica nanoparticles with a mean diameter of around $350 \mathrm{~nm}$. The solid stober spheres were successfully converted into homogeneous hollow spheres when simply treated with $\mathrm{Na}_{2} \mathrm{CO}_{3}$ in the presence of CTAB at an appropriate concentration. The energy dispersive spectrum on the SEM was used to perform elemental analysis on the mesoporous silica nanoparticles. The EDS spectrum of spherical nanoparticles prepared using CTAB as a pore generating agent is shown in Figure 6. The binding energies of $\mathrm{O}$ and $\mathrm{Si}$ are shown by the peaks around $0.5 \mathrm{kV}$ and $1.9 \mathrm{kV}$, respectively. Silica makes up 23.59 percent of the total weight. The EDS spectrum's remaining lines correspond to the other elements $(\mathrm{Na}, \mathrm{K}$, and $\mathrm{Al})$.

6.2.4. Drug Loading on Mesoporous Silica Nanoparticle. Sample extract and mesoporous silica nanoparticles stirred 24 hrs. Mesoporous silica nanoparticles are used to load samples or drugs onto honey comps. It is employed in further pharmacological treatment investigations in zebrafish [17].

6.3. Acute Toxicity Study. Acute toxicity study of a test substance is employed as an excellent tool for assessing its toxicity risk in the human health and the environment [18]. The results of acute toxicity studies indicate that M.charantia did not cause any adverse behavioral changes or mortality upon treatment. At the end of the study, all fishes were sacrificed, and the organ was collected and subjected to histopathological analysis.

6.4. Histopathological Examination of Tissues. The histopathological analysis was done for the organ (brain) of the fishes subjected to acute toxicity studies. Fishes from six different groups (group 1-control, group 2-(day 1) trimethyltin chloride, group 3-(day 2 trimethyltin chloride), group 4trimethyltinchloride + FDA approved drug (donepezil), group 5-trimethyltin chloride + charantin, group 6trimethyltin chloride + (charantin + mesoporous silica nanoparticle) were the organs were collected. Histopathology sections are viewed under $\times 40$ and $\times 40$ magnification photomicrograph (Figures 7-12).

6.4.1. Group I: Brain (Control). The section shows brain tissue with four layers, undamaged structure, and cellular morphology.

6.4.2. Group II: Brain (Day 1: Trimethyltin Chloride). The section shows cerebellar tissue with areas of degeneration, microinfarcts, the proliferation of ganglion cells, and diffuse inflammation.

6.4.3. Group III: Brain (Day 2: [Trimethyltin Chloride]). The section shows normal cerebellar tissue with focal Purkinje cell proliferation, mild edema, and degenerative changes.

6.4.4. Group IV: Brain [Trimethyltin Chloride + FDA Approved Drug (Donepezil)]. The section shows thinnedout normal cerebellum, occasional prominent congested vessels, and neurofilaments tissue with focal very minimal degenerative changes.

6.4.5. Group V: Brain (Trimethyltin Chloride + Charantin). The section shows brain tissue with normal structure and cellular morphology with focal degenerative changes.

6.4.6. GroupVI: Brain [Trimethyltin Chloride + (Charantin + Mesoporous Silica Nanoparticle)]. The section shows brain tissue with thinning of Purkinje cell layer, mild edema, and preservation of rest of the normal brain tissue. The zebrafish brain was identified by the various significant studies done by Dr. Ezhilvizhi Alavandar (Pathologist), Kilpauk Medical College, Chennai, Tamilnadu.

6.5. Acetylcholinesterase Activity Assay. One of the various therapeutic options is the use of AChE inhibitors, which enhance the quantity of acetylcholine present in the synapses between cholinergic neurons. The AChE inhibitory activity of various concentrations $(10-50 \mathrm{~g} / \mathrm{mL})$ of ethanol extract of M.charantia is demonstrated in strategies to enhance cholinergic functions in the brain. The results (Figure13) demonstrated that ethanol extract showed inhibitory action that was concentration-dependent. The testing results show that at a concentration of $50 \mathrm{~g} / \mathrm{mL}$, the ethanol extract of M.charantia exhibited significant AChE assay activity (81 $\pm 5.86 \%)$ when compared to the standard drug $(86 \pm 7.75 \%)$. 
6.6. DPPH (2, 2-Diphenyl-1-Picrylhydrazyl) Radical Scavenging Activity. DPPH free radicals are very stable, and it is a widely used method for determining the antioxidant potential in less time. The capacity to scavenge was tested using different concentrations of aqueous extracts of $\mathrm{M}$. charantia and ascorbic acid as a standard antioxidant (positive control). The results of the assay indicate that the aqueous extract of M. charantia exhibited significant DPPH radical scavenging activity $(66 \pm 8.58 \%)$ at a concentration of $10 \mathrm{~g} / \mathrm{mL}$. As the concentration was raised, the percentage of inhibition went up as well ( $82 \pm 4.44$ percent) in $50 \mathrm{~g} / \mathrm{mL}$, as seen in Figure14.

6.7. Reducing Power Assay. The total reducing power assay was used to assess the antioxidant potential of $M$. charantia ethanol extract. The ability to scavenge was evaluated using different concentrations of aqueous extracts of M.charantia and ascorbic acid as a standard antioxidant (positive control). The results of the assay indicate that the aqueous extract of $M$. charantia exhibited significant total reducing power activity $(69 \pm 2.15)$ at a concentration of $10 \mathrm{~g} / \mathrm{mL}$. As the concentration was increased, the percentage of inhibition went up as well $(80 \pm 2.15)$ in $50 \mathrm{~g} / \mathrm{mL}$, as seen in Figure 15.

6.8. Total Antioxidant Assay. Total antioxidant activity is a widely used method for determining the antioxidant potential of natural compounds in a short period because it is relatively stable. The ability to scavenge was evaluated using various concentrations of ethanol extracts of $M$. charantia and ascorbic acid as a standard antioxidant (positive control). The results of the assay indicate that the ethanol extract of $M$. charantia exhibited significant total antioxidant activity $(0.168 \pm 0.00025 \%)$ at a concentration of $10 \mathrm{~g} / \mathrm{mL}$. When the extract concentration was increased, the mean was also raised $(0.0325 \pm 0.00026$ percent $)$ at $50 \mathrm{~g} / \mathrm{mL}$, as shown in Figure16.

6.9. Lipid Peroxidation Assay. The lipid peroxidation assay was used to assess the antioxidant potential of $M$. charantia ethanol extract. Malondialdehyde was used as a standard antioxidant, and various concentrations of ethanol extracts of $M$. charantia were used to evaluate the scavenging activity. The results of the assay suggest that the ethanol extract of $M$. charantia exhibited lipid peroxidation activity at a concentration of $10 \mathrm{~g} / \mathrm{mL}(69 \pm 1.313)$. The percentage of inhibition increased when the concentration was increased $(87 \pm 3.474)$ in $50 \mathrm{~g} / \mathrm{mL}$, as seen in Figure 17.

\section{Conclusion}

The preliminary phytochemical analysis of the aqueous extract of $M$. charantia revealed the presence of steroids, tannins, flavonoids, terpenoids, etc., The HPTLC analysis revealed the presence of 12 compounds of steroids with bioactive potentials. The bioactive compound was confirmed and chactertized by Fourier transform infrared spectroscopy. The antioxidant potential of crude aqueous extracts of $M$. charantia was assessed by DPPH (2, 2- diphenyl-1-picrylhydrazyl) radical scavenging assay, and the results suggest that the extract showed excellent antioxidant activity. The trimethyltin chloride was subjected to Alzheimer's studies using Danio rario. At the end of the Alzheimer's treatment study, the behavioral and mortality changes were assessed, and the results show that the extract did not cause any adverse effects on zebrafish, which suggests that the $M$. charantia is safe for consumption. The histopathological analysis was done for the organ (brain), and the results show that there are no pathological changes in tissues of the fish treated with the $M$. charantia extract. The effect of the ethanol extract of $M$. charantia was also analyzed by the cell viability test (MTT assay) in fibroblast (normal cell line), and the results revealed that the extract did not affect the functions of normal cells. Since the M. charantia showed higher antioxidant activity and anticholinesterase activity, upon further characterization and assessment, this could be a safe and potential drug candidate for neurotoxicity. In the future, the study can be developed to formulate a drug against neurotoxicity.

\section{Data Availability}

All data used to support the findings of this study are included within the article.

\section{Conflicts of Interest}

The authors declare that there are no conflicts of interest.

\section{Acknowledgments}

The authors are thankful to Sri Ranga Ramanuja Centre for Advanced Research in Sciences (SRRCARS) for providing facilities to carry out this research work. The authors are would like to thank to the Management of Sathyabama Institute of Science and Technology, Chennai, for their help and cooperation.

\section{References}

[1] F. D. Gomez, P. Apodaca, L. N. Holloway, K. H. Pannell, and M. M. Whalen, "Effect of a series of triorganotins on the immune function of human natural killer cells," Environmental Toxicology and Pharmacology, vol. 23, no. 1, pp. 18-24, 2007.

[2] X. Tang, X. Yang, G. Lai et al., "Mechanism underlying hypokalemia induced by trimethyltin chloride: inhibition of $\mathrm{H}^{+} / \mathrm{K}^{+}$ATPase in renal intercalated cells," Toxicology, vol. 271, no. 12, pp. 45-50, 2010.

[3] M. Liong, J. Lu, M. Kovochich et al., "Multifunctional inorganic nanoparticles for imaging, targeting, and drug delivery," ACS Nanotechnology, vol. 2, no. 5, pp. 889-896, 2008.

[4] M. J. Saary and R. A. House, "Preventable exposure to trimethyl tin chloride: a case report," Occupational Medicine, vol. 52, no. 4, pp. 227-230, 2002.

[5] Q. F. Zhou, G. B. Jiang, and J. Y. Liu, "Small-scale survey on the contamination status of butyltin compounds in seafoods collected from seven Chinese cities," Journal of Agricultural Food Chemistry, vol. 49, no. 9, pp. 4287-4291, 2001. 
[6] P. Ragavendran, D. Sophia, C. Arul Raj, and V. K. Gopalakrishnan, "Functional group analysis of various extracts of Aerva lanata (L.,) by FTIR spectrum," Pharmacology Online, vol. 1, pp. 358-364, 2011.

[7] S. Kwon, R. K. Singh, R. A. Perez, E. A. Abou Neel, H. W. Kim, and W. Chrzanowski, "Silica-based mesoporous nanoparticles for controlled drug delivery," Journal of Tissue Engineering, vol. 4, 2013.

[8] S. Jia, M. Shen, F. Zhang, and J. Xie, "Recent advances in Momordica charantia: functional components and biological activities," International Journal of Molecular Sciences, vol. 18, no. 12, p. 2555, 2017.

[9] S. Hemmalakshmi, S. Priyanga, and K. Devaki, "Phytochemical screening and HPTLC fingerprinting analysis of ethanolic extract of Erythrina variegata l. flowers," International Journal of Pharmacy and Pharmaceutical Sciences, vol. 8, pp. 210-217, 2016.

[10] T. Becker and C. G. Becker, "Axonal regeneration in zebrafish," Current Opinion in Neurobiology, vol. 27, pp. 186-191, 2014.

[11] C. Tourne-Peteilh, S. Begu, D. A. Lerner, A. Galarneau, U. Lafont, and J. M. Devoisselle, "Sol-gel one-pot synthesis in soft conditions of mesoporous silica materials ready for drug delivery system," Journal of Solgel Science and Technology, vol. 61, no. 3, pp. 455-462, 2012.

[12] J. B. Harborne, Textbook of phytochemical methods. A guide to modern techniques of plant analysis, Chapman and Hall Ltd, London, 5th Ed edition, 1998.

[13] F. Armetta, M. L. Saladino, C. Giordano et al., "Non-conventional Ce:YAG nanostructures via urea complexes," Scientific Reports, vol. 9, no. 1, p. 3368, 2019.

[14] S. K. Srivastava, S. Trajmar, A. Chutjian, and W. Williams, "Absolute elastic differential electron scattering cross sections in the intermediate energy region. III. SF6and UF6," The Journal of Chemical Physics, vol. 64, no. 7, pp. 2767-2771, 1976.

[15] T. S. Vijayakumar, S. Mahboob, G. Bupesh et al., "Facile synthesis and biophysical characterization of egg albumenwrapped zinc oxide nanoparticles: a potential drug delivery vehicles for anticancer therapy," Journal of Drug Delivery Science and Technology, vol. 60, article 102015, 2020.

[16] N. J. Borges, R. S. Manuel, C. L. Elam, and B. J. Jones, "Differences in motives between millennial and generation X medical students," Medical Education, vol. 44, no. 6, pp. 570-576, 2010.

[17] J. Kim, J. Shim, S. Lee et al., "Rg3-enriched ginseng extract ameliorates scopolamine-induced learning deficits in mice," BMC Complementary and Alternative Medicine, vol. 16, p. 66, 2012.

[18] A. Braca, N. de Tommasi, L. di Bari, C. Pizza, M. Politi, and I. Morelli, "Antioxidant Principles fromBauhiniatarapotensis," Journal of Natural Products, vol. 64, no. 7, pp. 892-895, 2001. 